\title{
Effects of observation cost on sequential search behavior'
}

\author{
AMNON RAPOPORT ${ }^{2}$ \\ UNIVERSITY OF NORTH CAROLINA
}

\begin{abstract}
Sixty Ss participated individually in a search problem attempting to find a single target object located in one of several distinguishable regions. The search consisted of sampling observations with replucement from the various regions. Ss were told the prior probability of finding the target in a given region, the conditional miss probability that if the target is in a given region it will not be detected on a single observation there, and the cost per observation for each region. The effect of observation costs was investigated. Procedures devised for comparing actual and optimal search behavior showed that relative to the optimal policy $S$ s erred in the direction of maximizing detection probabilities rather than in the direction of minimizing the cost per observation, and that this error increased in magnitude the larger the differences among observation costs. A Markov model was tested and rejected. Implications of the study to visual search problems are discussed briefly.
\end{abstract}

Sequential decision-making processes operating in search behavior have been recently studied experimentally within the context of optional stopping problems (Kahan, Rapoport, \& Jones, 1967; Rapoport \& Tversky, 1966, 1969). Independent and identically distributed random variables could be observed sequentially in these studies at a fixed cost per observation. The $S$ could stop the search at a given stage and receive a payoff that was a function of the values of the observations taken up to that stage or he could continue the search for another observation (with a possibly higher value). Rapoport and Tversky (1966) studied the case where the distribution of observations was assumed to be known by $S$ s and the number of observations that could be taken was unbounded. In a second study of Rapoport and Tversky (1969) choice behavior was investigated under two different payoff functions where the number of observations that could be taken was bounded and small. Kahan et al (1967) studied a different case where the distribution of observations was initially unknown to $\mathrm{S}$, observations were costless, and the number of observations that could be taken was bounded and known. Most of the $S s$ who participated in these experiments behaved optimally.

The presentation order of observations in optional stopping tasks, whether random or not, cannot be affected by S's behavior. It is either predetermined by the $E$ or by some chance mechanism. In many other, more general, sequential search problems it is the decision maker rather than nature who has complete control of the sequence by which observations are taken; he decides at each stage where to search. The present study is concerned with such a sequential search problem in which an object is to be found that is located in one of several distinguishable places, the sequence of observations taken is determined by $S$, there is a probability of missing the object when the location in which it is hidden is being searched, and cost of the search differs from one stage to another, depending on the particular location searched. Several variants of this search problem can be distinguished, depending on the number of objects to be found, the form of the cost function, and whether or not the object (or objects) can move during the search period from one location to another. This study employs a search problem with only one object that cannot move. The problem is described more formally as follows.
A single target object that cannot move is located in one of a finite set, $R$, of possible locations. The $R$ locations may be searched one at a time, and it is assumed that the outcomes of the search are independent, conditional only on the inspection procedure used and the location of the object. The search terminates when the object is found. A prior probability $\mathrm{p}_{\mathrm{i}}$ that the object is in location $i, i=1,2, \cdots, R$, is assumed to be known

$$
\left(\sum_{i=1}^{R} p_{i}=1\right),
$$

as well as a fixed conditional miss probability $m_{i}\left(0<m_{i}<1\right)$ that if the object is in location $i$ it will not be detected on a single look there. Associated with each location $i$ is a cost, $c_{i}$, for a single look there. The $S$ 's objective is to find an inspection policy that minimizes the expected cost of the search.

Several search tasks can be modeled by the above search paradigm. Flying over a large area and searching for a camouflaged target such as a missile base may serve as one example. The values of $p_{i}$ in this example are determined by military intelligence information previously acquired about the target and its possible location, the values of $\mathrm{m}_{\mathrm{i}}$ are determined by the landscape of $\mathrm{R}$ different distinguishable regions in the area, and the values of $c_{i}$ may reflect the distances to be flown in order to reach each of the different regions, the risks taken in flying over them, etc. Looking for the key to your car that was hidden by your child in one of several rooms may serve as another suggestive example, where cost of the search may be expressed in terms of effort, time spent in the search, or aggravation.

The mathematical aspects of this search problem have been studied by Blackwell (see Matula, 1964), who used dynamic programming, and Black (1965), who used a simpler, more transparent approach. They found the optimal search policy for the problem that minimizes the expected cost of the search and consists of an infinite sequence indicating the location to be searched at any stage. Matula (1964), who was concerned with the question of periodic features of the optimal policy, found necessary and sufficient conditions ensuring ultimate periodicity. Chew (1967) attempted to answer a similar question when the target was in one of $R+1$ locations but searching was permitted only among the first $R$, i.e., when

$$
\sum_{i=1}^{R} p_{i}<1 .
$$

The optimal search policy will be described in a subsequent section. It is unrealistic, of course, to expect the optimal policy to provide an exact account of Ss' search behavior, though it may provide a good first approximation. The main purpose of this study is to devise and apply procedures for detecting discrepancies from optimal search behavior that may be interpreted psychologically and may reveal the effects of observation cost and detection probability on Ss' choice behavior. If these discrepancies are found to be large in magnitude and highly systematic, certain constraints would need to be incorporated in the optimal model or other models would have to 
be constructed to describe adequately the observed choices. A secondary purpose of this study is to discuss briefly the relevance of the present search paradigm to studies of visual search.

\section{METHOD}

Subjects

The Ss were 60 male undergraduate students at the Hebrew University of Jerusalem, who volunteered to participate in a paid decision-making experiment. Twenty Ss were assigned to each of three experimental groups, 1,2 , and 3 . Ss were run individually for one session that lasted approximately $1 \mathrm{~h}$.

\section{Apparatus}

A vertical $36 \times 23$ in. board was mounted on a table about $14 \mathrm{in.}$ in front of $\mathrm{S}$, separating him from the E. Four round holes, $3 \frac{1}{2}$ in. apart horizontally, were made in the board, 8 in. above the table. Behind each hole, on the other side of the board facing the $E$, was a $4 \times 4 \times 6$ in. plastic box with marbles of uniform size and same color. $S$ could not see the marbles in the boxes but could push his hand easily through any of the four holes, pick up any marble and observe its color. Above each hole on both sides of the board was written the box number $\mathrm{i}(\mathrm{i}=\mathrm{A}, \mathrm{B}, \mathrm{C}, \mathrm{D})$, as well as the number of marbles in the box, and the cost (in points), $c_{i}$, for observing a single marble in Boxi. The $E$ was seated across the table, facing the boxes and recording $S$ 's decisions.

\section{Procedure}

Ss were told that the experiment consisted of several independent problems. At the beginning of each problem the $\mathbf{E}$ would select a box and replace one of its marbles with the target marble ..a marble of the same size and weight but of a different color. $S$ was told that the location of the target on each problem was predetermined randomly, with equal probability of one-fourth for each box, and that the location would not be affected by his decisions or the locations of the target in the preceding problems.

An initial sum of 50 points for Group 1, 100 points for Group 2, and 200 points for Group 3 was given to $S$ at the beginning of each problem. On each trial $S$ put his hand through one of the holes, picked up one of the well-mixed marbles, and observed its color. If this was the target marble the problem was solved. Otherwise, $S$ dropped the marble back in its box and moved to the next trial, after the $E$ recorded and announced the cost, $c_{;}$. for the observation and the accumulated cost of the search for the problem. The search was terminated either when $S$ lost his starting capital for a given problem or when he picked up the target marble. In the latter case he was paid one agora (Groups I and 3) or two agorot (Group 2) for every point he gained. (One agora equals .286థ.) $\mathrm{S}$ was instructed to maximize his gain for every problem (i.e., minimize the cost of search).

The starting capital and the observation costs (presented in Table 1) were so adjusted that the mean number of looks (observations) per problem would be approximately equal for the three groups. The mean number of looks per problem was $27.781,24.769$, and 24.350 for Groups 1,2 , and 3 , respectively, with one-way analysis of varjance yielding no significant differences among groups, $F(2,57)=1.59, \mathrm{p}>.1$.

Every $S$ participated in eight problems with prior probabilities $p_{i}=1 / 4$ for all $i$. The number of marbles in each box, the values of $c_{i}$, and the conditional miss probabilities, $m_{i}$, are presented in Table 1. The groups differed from one another in the values of $c_{i}$. In Group 1 it cost one point to look (observe the color of just one marble) in any of the boxes, while in Groups 2 and 3 there was an inverse relationship between $m_{i}$ and $c_{i}$.

The target marble was located twice in Box A, three times in
Table 1

Summary of Information Provided to Ss

\begin{tabular}{|c|c|c|c|c|}
\hline & \multicolumn{4}{|c|}{$\mathrm{BOx}$} \\
\hline & A & B & $\mathrm{C}$ & $\mathrm{D}$ \\
\hline Number of marbles & 4 & 7 & 13 & 19 \\
\hline $\mathrm{m}_{\mathrm{i}}$ & 750 & .857 & .923 & .947 \\
\hline$c_{i}$ (group 1) & 1 & 1 & 1 & 1 \\
\hline$c_{i}$ (group 2$)$ & 4 & 3 & 2 & 1 \\
\hline$c_{i}$ (group 3) & 20 & 10 & 5 & 1 \\
\hline
\end{tabular}

Box B, once in Box C, and twice in Box D. The order of presentation of problems was varied randomly subject to the constraint that the same box did not appear on successive problems.

\section{The Optimal Policy}

\section{RESULTS}

An application of Bayes's theorem shows that the optimal policy minimizing expected cost of the search is to always look in the box for which the posterior probability (given the failure of the preceding looks prescribed by the optimal policy) of finding the target marble divided by the cost per look is highest. Consider all posterior probabilities of finding the target marble (hereafter the detection probabilities) divided by the cost per look, which have the form

$$
p_{i}\left(1-m_{i}\right) m_{i}^{n-1} / c_{j}
$$

arranged in a two-dimensional $n$ by $\mathrm{i}$ array. The optimal policy (Black, 1965) is to look first in the box corresponding to the column that includes the largest entry in the array, then look in the box corresponding to the column including the next largest entry in the array, etc. The optimal policy thus rearranges the entries of the array by size. The resulting sequence of entries will be referred to as the optimal sequence and denoted by $G_{v}=\left(g_{v} 1\right.$, $\left.\mathrm{g}_{\mathrm{v}}, \cdots\right)$, where the subscript $v$ denotes the group, j denotes the rank of the element in the sequence, $j=1,2, \cdots$, and $g_{v j} \geqslant g v, j+1$. The first 50 looks y ielded by the optimal policy are presented in Table 2 for Groups 1, 2, and 3

Table 2

The First 50 Optimal Decisions for Groups 1, 2, and 3

\begin{tabular}{|c|c|c|c|c|c|c|c|}
\hline \multirow[b]{2}{*}{ Trial } & \multicolumn{3}{|c|}{ Group } & \multirow[b]{2}{*}{ Trial } & \multicolumn{3}{|c|}{ Group } \\
\hline & 1 & 2 & 3 & & 1 & 2 & 3 \\
\hline 1 & $\mathrm{~A}$ & A & $D$ & 26 & $\mathbf{A}$ & A & $\mathrm{D}$ \\
\hline 2 & A & $D$ & $D$ & 27 & C & $\mathrm{D}$ & B \\
\hline 3 & B & $\mathrm{D}$ & $\mathrm{D}$ & 28 & $\mathrm{D}$ & $\mathrm{C}$ & $\mathrm{C}$ \\
\hline 4 & $A$ & B & $\mathrm{D}$ & 29 & B & B & D \\
\hline 5 & B & D & $\mathrm{D}$ & 30 & C & $\mathrm{D}$ & C \\
\hline 6 & A & A & $\mathrm{D}$ & 31 & D & $\mathrm{C}$ & D \\
\hline 7 & B & $\mathrm{D}$ & $\mathrm{D}$ & 32 & D & $\mathrm{D}$ & A \\
\hline 8 & B & $\mathrm{D}$ & D & 33 & $\mathrm{C}$ & D & B \\
\hline 9 & A & B & $\bar{D}$ & 34 & D & B & $\mathrm{D}$ \\
\hline 10 & B & D & D & 35 & B & $\mathrm{C}$ & C \\
\hline 11 & $C$ & $\mathrm{C}$ & $\mathrm{D}$ & 36 & $\mathrm{C}$ & D & D \\
\hline 12 & $C$ & $\mathrm{D}$ & D & 37 & D & C & C \\
\hline 13 & B & $\mathrm{D}$ & $\mathrm{D}$ & 38 & A & D & $\mathrm{D}$ \\
\hline 14 & $C$ & C & D & 39 & D & A & B \\
\hline 15 & $\mathrm{C}$ & A & D & 40 & $C$ & B & D \\
\hline 16 & A & B & $\mathrm{D}$ & 41 & D & $\mathrm{D}$ & C \\
\hline 17 & $B$ & D & $\mathrm{D}$ & 42 & B & $C$ & $\mathrm{D}$ \\
\hline 18 & $C$ & C & $\mathrm{D}$ & 43 & $C$ & $\mathrm{D}$ & C \\
\hline 19 & $\mathrm{D}$ & D & $\bar{D}$ & 44 & D & $C$ & A \\
\hline 20 & C & D & $\mathrm{D}$ & 45 & D & D & D \\
\hline 21 & D & C & $\mathrm{D}$ & 46 & C & $\bar{B}$ & B \\
\hline 22 & B & B & $\mathrm{D}$ & 47 & B & D & D \\
\hline 23 & $\mathrm{C}$ & D & $\mathrm{D}$ & 48 & $\mathrm{D}$ & C & C \\
\hline 24 & $\mathrm{D}$ & C. & $\mathrm{C}$ & 49 & C & D & D \\
\hline 25 & D & D & D & 50 & A & A & C \\
\hline
\end{tabular}


Table 3

Proportions of First Observations Made in Box

\begin{tabular}{ccccc}
\hline & \multicolumn{3}{c}{ Box } & \\
\cline { 2 - 5 } Group & $\mathrm{A}$ & $\mathrm{B}$ & $\mathrm{C}$ & $\mathrm{D}$ \\
\hline 1 & .650 & .169 & .125 & .056 \\
2 & .537 & .181 & .144 & .138 \\
3 & .250 & .244 & .194 & .312 \\
\hline
\end{tabular}

To behave optimally, Ss in Groups 1 and 2 should make their first look on every problem in Box A, while Ss in Group 3 should first look in Box D. Table 3 shows the proportion of first observations made in every box, summed over all eight problems and $20 \mathrm{Ss}$ in each group. The table shows that the first observation was made optimally by the majority of the Ss in Groups 1 and 2, and that the proportions of optimal first observations decreased over groups. The null hypothesis of equal proportions for each group (i.e., equal proportions in each column of Table 3) was rejected, $\chi^{2}(12)=66.16, p<.001$. Similar results were obtained when independence between problems was not assumed and the chi-square test was applied to each problem separately.

Every $\mathrm{S}$ made $\mathrm{N}_{\mathrm{w}}$ looks in problem $\mathrm{w}, \mathrm{w}=1,2, \cdots, 8$. Considering a sequence of $\mathrm{N}_{\mathrm{w}}$ looks, a number of the form (1) can be computed, yielding for each trial the detection probability (given the failure of S's earlier looks in problem w) divided by the cost per look. The sequence of numbers of the form (1), computed from the observed $\mathrm{N}_{\mathrm{w}}$ looks of a particular $\mathrm{S}$, will be called the observed sequence and denoted by $R_{v w}=\left(r_{v 1}, r_{v 2}\right.$, $\cdots, \mathrm{r}_{\mathrm{v}} \mathrm{N}_{\mathrm{w}}$ ). The elements of $\mathrm{R}_{\mathrm{vw}}$ will typically not be ordered by size. $r_{v j} \geqslant r_{v, j+1}$ if and only if $r_{v j}=g_{v j}$ for all $j, j=1,2, \cdots, N_{w}$. Otherwise, $r_{v j} \gtrless r_{v, j+1}$.

Let $\mathrm{G}^{*}{ }_{\mathrm{vw}}=\left(\mathrm{g}^{*}{ }_{\mathrm{v} 1}, \mathrm{~g}^{*}{ }_{\mathrm{v} 2}, \cdots, \mathrm{g}^{*} \mathrm{vN}_{\mathrm{w}}\right)$ denote the cumulative optimal sequence, where

$$
g^{*}{ }_{v j}=\sum_{t=1}^{j} g_{v t}
$$

Similarly, let $R^{*}{ }_{v w}=\left(r^{*}{ }_{v 1}, r^{*}{ }_{v .2}, \cdots, r^{*}{ }_{v N_{w}}\right)$ denote the cumulative observed sequence for a given $\mathrm{S}$ and a given problem w, where

$$
r_{v j}^{*}=\sum_{t=1}^{j} I_{v t} .
$$

Clearly, as $\mathbf{G}_{v w}$ is the cumulative optimal sequence, $g^{*}{ }_{v j} \geqslant r^{*}{ }_{v j}$. Define $\alpha_{v j}=g^{*} v j-r^{*}{ }_{v j}, \alpha_{v j} \geqslant 0$. A measure of discrepancy from optimal search behavior is given by

$$
\mathrm{z}_{\mathrm{vw}}=\sum_{\mathrm{j}=1}^{\mathrm{N}_{w}} \alpha_{\mathrm{vj}} / \mathrm{N}_{w},
$$

which can be computed for every $S$ and every problem $w$ in group $v$.

The values of $\mathrm{z}_{\mathrm{vw}}$ depend on the detection probabilities and observation costs appearing in the equations for the optimal and observed sequences. In general, the higher the values of $c_{i}$ the smaller the value of $z_{\mathrm{vw}}$. Hence, when sequential search tasks differ from one another in their cost structure, $\mathrm{z}_{\mathrm{vw}}$ cannot be used for comparing these tasks with one another in terms of the discrepancy between actual and optimal search behavior. The values of $\mathrm{z}_{\mathrm{vw}}$ can be used only to compare sequential search tasks having the same observation costs, or to test for problem effect within the same task.
The values of $z_{\mathrm{vw}}$ were computed for every $S$ and every problem. One-way analysis of variance with repeated measures was carried out for each group to test for a problem order effect. The results were nonsignificant in each case, $F(7,113)=1.87$, $\mathrm{p}>.1, \mathrm{~F}(7,113)=1.23, \mathrm{p}>.25$, and $\mathrm{F}(7,113)=2.08, \mathrm{p}>.05$, for Groups 1, 2, and 3, respectively. Thus, for each group there is no indication of problem-to-problem learning in terms of the size of discrepancy from optimal search behavior.

The optimal policy is determined by the detection probabilities and observation costs. To behave optimally, $S$ should balance optimally two objectives: (1) maximizing the detection probability, and (2) keeping to minimum the cost of each observation. Rarely, as for instance in Group 1, these two objectives will be consistent with each other, since the policy that maximizes detection probability in Group 1 minimizes simultaneously the cost of each look and provides the optimal solution for the problem. More frequently, when the observation cost depends on the region searched, the two goals cannot be satisfied simultaneously. Thus, the policy that maximizes the detection probability for Groups 2 and 3 (i.e., the optimal policy for Group 1) and the policy that minimizes the cost of each look (sampling from Box D on every trial) differ from each other and also from the optimal policies for these groups.

Figure 1 portrays the cumulative detection probabijities as a function of the number of looks. The upper curve corresponds to the policy maximizing the detection probability. Since $c_{i}=1$ for all $i$ in Group 1 this is the optimal policy for Group 1 . The second and third curves from the top show the cumulative detection probabilities for the optimal policies (see Table 2) for Groups 2 and 3 , respectively. The lower curve portrays the cumulative detection probability when Box D is searched on every trial. Nonoptimal search behavior in Groups 2 and 3 would result from weighing too heavily one of the two objectives relative to the optimal balancing. By comparing the cumulative detection probability curve resulting from the observed looks of a certain $S$ with the second curve (for Ss in Group 2) or the third curve (for Ss in Group 3) from the top, the nature and size of certain biases affecting his search behavior can be assessed.

Consider the detection probabilities

$$
p_{i}\left(1-m_{i}\right) m_{i}^{n-1}
$$

arranged in a two-dimensional $n$ by $i$ array, and let $P_{v}=\left(p_{v 1}, p_{v 2}\right.$, ...) denote the optimal detection sequence of entries from this array corresponding to the optimal sequence $G_{v}$. That is, $P_{v}$ is obtained from $G_{v}$ by dividing each element $g_{v j}$ by the cost per observation on trial $j$. Clearly, $P_{1}=G_{1}$ since $c_{i}=1$ for all $i$ in Group 1 , but $P_{2} \neq G_{2}$ and $P_{3} \neq G_{3}$. Similarly, let $Q_{v w}=\left(q_{v 1}\right.$, $\mathrm{q}_{\mathrm{v} 2}, \cdots, \mathrm{q}_{\mathrm{vN}}$ ) denote the observed detection sequence corresponding to the observed sequence $R_{v w}$. That is, $Q_{v w}$ is obtained from $R_{v w}$ by dividing $r_{v j}$ by the cost per observation made by $S$ on trial $j$ of problem $w$. Let $Q^{*}{ }_{v w}=\left(q^{*} v_{1}, q^{*} v_{2}, \cdots\right.$, $\mathrm{q}^{*} \mathrm{vN}_{\mathrm{w}}$ ) denote the cumulative observed detection sequence, where

$$
q_{v j}^{*}=\sum_{t=1}^{j} q_{v t},
$$

and similarly, $\mathrm{P}^{*}{ }_{\mathrm{vw}}=\left(\mathrm{p}^{*}{ }_{\mathrm{v} 1}, \mathrm{p}^{*}{ }_{\mathrm{v} 2}, \cdots, \mathrm{p}^{*} \mathrm{vN}_{\mathrm{w}}\right)$ denote the cumulative optimal detection sequence, where

$$
p^{*}{ }_{v j}=\sum_{t=1}^{j} p_{v t} .
$$

A measure of discrepancy from cumulative optimal detection probability is given by 


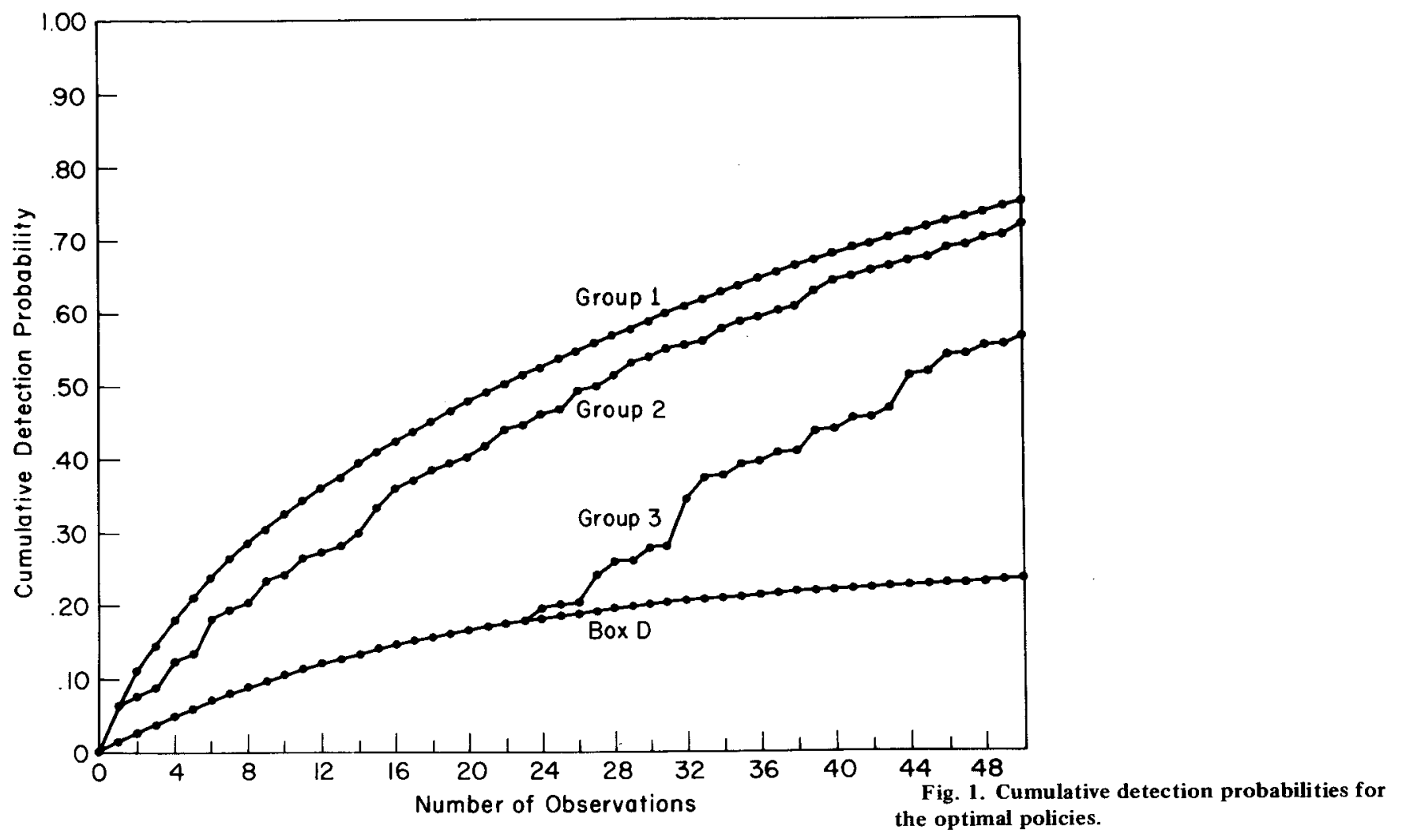

$$
y_{v w}=\sum_{j=1}^{N_{w}} \beta_{v j} / N_{w},-1<y_{v w}<1,
$$

where $\beta_{v j}=p^{*}{ }_{v j} \cdots q^{*}{ }_{v j}$. Since $p_{1 j}=g_{1 j}$ and $q_{1 j}=r_{1 j}$, then $y_{1 w}=z_{1 w}$. However, $y_{2 w} \neq z_{2 w}$ and $y_{3 w} \neq z_{3 w}$. A positive value of $y_{v w}$ means that the optimal balancing between maximizing detection probability and minimizing the cost of each observation has not been achieved, but that $\mathrm{S}$ was biased toward the second objective. A negative value of $\mathrm{y}_{\mathrm{vw}}$ indicates the opposite bias.

The means and SDs of $y_{v w}$, computed over Ss for each problem separately, are presented in Table 4 for Groups 2 and 3. Inspection of the table reveals that for all but one problem (Problem 8 of Group 2) the means of $y_{v w}$ are negative, and that they are considerably more negative for Group 3 than for Group 2. Relative to the optimal policy Ss were more concerned in their search behavior with maximizing the posterior probability of finding the target marble than in minimizing the cost per observation. This bias had only a small effect in Group 2 but a much stronger effect in Group 3, where differences among the values of $c_{i}$ were much larger.

Of special signficance are the SDs reported in Table 4. They show that even in Group 2, where the mean values of $y_{2} w$ were very close to zero (the mean difference between $q^{*} 2 w$ and $p^{*} 2 w$ was about one percentage point), individual search behavior was typically nonoptimal. Several Ss showed a strong bias for maximizing detection probability while other Ss showed a strong opposite bias. In Group 3 almost all the Ss showed a bias for maximizing detection probability (the mean difference between $\mathrm{q}^{*} 3 \mathrm{w}$ and $\mathrm{p}^{*} 3 \mathrm{w}$ was about nine percentage points), but there were considerable individual differences in the size of these biases.

\section{Stepwise Optimal Looks}

The analysis of the $z_{v w}$ and $y_{v w}$ measures is only a first step in the comparison of actual with optimal search behavior. It can indicate problem-to-problem learning effects and biases influencing $S$ 's search behavior but it sheds little light on the amount of nonoptimality and the type of errors Ss made. The simplest way to assess the amount of nonoptimality might seem to compute for each $S$ the proportion of optimal looks he made in all eight problems. This computation, however, is hard to interpret since to determine the optimal observation for stage $j+1$ the optimality of observations 1 through $j$ has to be assumed. What is required is a model that, when provided with the actual $\mathrm{j}$ looks of a given $\mathrm{S}$, prescribes the stepwise optimal look for state $\mathrm{j}+1$, without assuming optimality of the immediately preceding $\mathrm{j}$ looks.

The stepwise optimal policy prescribes to look always in the box for which the posterior probability (given the failure of S's earlier looks in a certain problem) of finding the target marble divided by the cost per look is highest. Clearly, the stepwise

Table 4

Means and Standard Deviations of Discrepancies from Cumulative Optimal Detection Probability

\begin{tabular}{|c|c|c|c|c|}
\hline \multirow[b]{2}{*}{ Problem } & \multicolumn{2}{|c|}{ Group 2} & \multicolumn{2}{|c|}{ Group 3} \\
\hline & Mean & $\mathrm{SD}$ & Mean & SD \\
\hline 1 & -.0163 & .0298 & -.1336 & .0613 \\
\hline 2 & -.0005 & .0451 & -.1167 & .0687 \\
\hline 3 & -.0038 & .0351 & -.0887 & .0721 \\
\hline 4 & -.0228 & .0243 & -.0273 & .0778 \\
\hline 5 & -.0061 & .0535 & -.1017 & .0735 \\
\hline 6 & -.0013 & .0565 & -.0949 & .0911 \\
\hline 7 & -.0045 & .0310 & -.0806 & .0744 \\
\hline 8 & .0013 & .0685 & -.0810 & .0681 \\
\hline Mean & -.0067 & .0430 & -.0906 & .0734 \\
\hline
\end{tabular}


Table 5

Means and Standard Deviations of Proportions of Stepwise Optimal Observations

Box

\begin{tabular}{lccccc} 
Group & A & B & C & D & Total \\
\hline l Mean & .518 & .283 & .113 & .168 & .220 \\
SD & .219 & .142 & .105 & .301 & .063 \\
2 Mean & .290 & .049 & .009 & .618 & .227 \\
SD & .117 & .056 & .016 & .249 & .074 \\
3 Mean & .010 & .020 & .011 & .938 & .430 \\
SD & .033 & .035 & .026 & .112 & .087 \\
\hline
\end{tabular}

optimal observations have to be computed separately for every $S$ and every problem. They will correspond to the optimal observations for a certain problem if $S$ never deviates from the optimal policy on this problem.

A computer program was written to obtain for every $S$ and every problem the stepwise optimal sequence of observations, and to compute the proportion of S's observations that were actually stepwise optimal. Let $\mathbf{d}_{\mathbf{i k}}$ denote the number of looks in Box $\mathrm{i}$ when Box $k$ should be observed by the stepwise optimal policy, summed over all the observations made by a given $S$ in all eight problems, Let

$$
\mathrm{h}_{\mathrm{ik}}=\mathrm{d}_{\mathrm{ik}} / \sum_{\mathrm{k}} \mathrm{d}_{\mathrm{ik}} \text {, and } \mathrm{h}_{\mathrm{i}}=\sum_{\mathrm{i}} \mathrm{d}_{\mathrm{ii}} / \sum_{\mathrm{i}} \sum_{\mathrm{k}} \mathrm{d}_{\mathrm{ik}}
$$

$h_{\mathrm{ii}}$ denotes the proportion of looks in Box $\mathrm{i}$ that were stepwise optimal, and $h_{i}$ denotes the proportion of all the observations made by $S$ (summed over the four boxes and eight problems) that were stepwise optimal. The means and $\mathrm{SDs}$ of $\mathrm{h}_{\mathrm{ii}}, \overline{\mathrm{h}}_{\mathrm{ii}}$, and $S D\left(h_{i i}\right)$, respectively, are presented in the first four columns of Table 5. The means and SDs of $h_{j}$ are shown on the last column of this table.

Inspection of Table 5 shows that the mean proportion of stepwise optimal observations was about .22 for Groups 1 and 2, and .43 for Group 3. The differences between the three means were significant, $F(3,357)=47.6, p<.001$. While there were no significant differences between the mean proportions of Groups 1 and 2 , the mean proportion of Group 3 was significantly larger than both. The table also shows considerable differences in the values of $\bar{h}_{\mathrm{ii}}$ between and within groups. Since the distributions of $h_{i i}$ were typically skewed and frequently bimodal and the variances of these distributions varied considerably from one another (see Table 5) an ordinal analysis of the data was made. The Friedman analysis of variance by ranks test (Siegel, 1956, p. 166) was applied to the ranks of the values of $h_{i i}$ for each group separately. The Box effect was significant at the .001 level for all three groups, $\chi_{\mathrm{r}}^{2}(3)=27.12, \chi_{\mathrm{r}}^{2}(3)=52.12$, and $\chi_{\mathrm{r}}^{2}(3)=36.28$ for Groups 1,2 , and 3 , respectively.

A comparison of the values of $\overline{\mathrm{h}}_{\mathrm{ii}}$ across groups shows that $\overline{\mathrm{h}}_{\mathrm{AA}}, \overline{\mathrm{h}}_{\mathrm{BB}}$, and $\overline{\mathrm{h}}_{C \mathrm{C}}$ decreased over groups, while $\overline{\mathrm{h}}_{\mathrm{DD}}$ showed the opposite trend. $\bar{h}_{D D}$ assumed the value of .168 for Group 1 and reached the high value of .938 for Group 3 , while the values of $\bar{h}_{A A}, \bar{h}_{B B}$, and $\bar{h}_{C C}$ in Group 3 were practically zero. The results reported in Table 5 suggest that $h_{\mathrm{ii}}$ is a function of both $m_{i}$ and $c_{i}$. When $c_{i}=1$ for all $i$, as in Group 1 , then the smaller the value of $m_{i}$ the higher the value of $h_{i i}$. (The one reversal $\bar{h}_{D D}>\bar{h}_{C C}$ for Group 1 is explained by the fact that $h_{D D}=1.0$ for three $S s$ in this group, and these three proportions were based in each case on only one observation.) As discrepancies among the values of $c_{i}$ increase, the effect of $m_{i}$ on $h_{i i}$ decreases and the proportion of stepwise optimal observations of the cheapest box iii _eases. This interpretation is consistent with the fact that the relation $\bar{h}_{A \mathrm{~A}}>\overline{\mathrm{h}}_{\mathrm{BB}}>\overline{\mathrm{h}}_{\mathrm{CC}}$, which is very strong in Group 1 , still appears in Group 2, but disappears in Group 3, where the discrepancies among the values of $c_{i}$ are the largest. At the same time the value of $\bar{h}_{D D}$ increases steadily across groups.

\section{A Markov Model}

Search behavior has been studied in the preceding sections relative to a normative model that prescribes how Ss should behave if they wish to minimize the expected cost of the search. The model has failed to provide an adequate description of the observed search behavior. Furthermore, though the analysis has indicated the nature and size of biases affecting Ss' behavior, it has not suggested how to utilize these biases effectively for constructing a suboptimal model for describing Ss' behavior. An alternative approach, unconcerned with optimality questions, is to construct and test a variety of descriptive models. The characteristic features of the present experiment-known costs and fixed conditional miss probabilities, discrete trials, and a relatively small number of locations where the target may be placed-as well as the success of Markov models for relatively simple tasks (Atkinson, Bower, \& Crothers, 1965) suggest an ergodic Markov chain model as the most likely candidate.

The model assumes that the sequence of observations made by the $\mathrm{Ss}$ is a finite-state ergodic Markov chain generated by a transition matrix with observable states. The transition probabilities are assumed to be independent of the trial number and to assume the same values for all eight problems for a given $S$. For terminology and complete treatment of finite Markov chains see Kemeny and Snell (1960).

No attempt is being made at this stage to formulate the Markov model in terms of more basic axioms, which offer an account of the psychological processes that would lead Ss to exhibit the pattern of search behavior observed in the present experiment. Even the order of the transition matrix is not being specified yet. A confirmation of the model's predictions might suggest how to identify the states and parameters of the model and derive them from a psychological theory of sequential search processes.

In analyzing the actual sequences of looks for Markov chain properties, standard tests (Anderson \& Goodman, 1957), which have been extensively used by Suppes and Atkinson (1960), will be used. Stationarity of the transition probabilities will be assumed and not tested for because of the relatively small number of trials in each problem and because of the results reported in the preceding sections showing no learning over problems. The first test concerns the null hypothesis that observations are statistically independent (zero-order process) against the alternative hypothesis that the process is a first-order Markov chain. If the first null hypothesis is rejected a second null hypothesis in testing the order of the chain is that the process is a first-order Markov chain against the alternative hypothesis that it is a second-order chain. Rejection of the second null hypothesis would mean that the probability of looking in a certain box can be predicted better by observing the two immediately preceding looks rather than the single immediately preceding look.

The transition matrices of the first-order Markov chain are presented in Table 6 for each group. The transition probabilities were computed over the eight problems and $20 \mathrm{Ss}$ in each group. The high values that appear on the main diagonal suggest immediately that the first null hypothesis is false. Indeed, assuming homogeneity of the individual Markov chains, the first hypothesis was tested by chi-square and rejected for all three groups at the .001 level. The hypothesis was then tested independently on the transition matrices of each $S$ and rejected for all 60 Ss. These results indicate that the prediction of search behavior is significantly improved if one knows the observation on the preceding trial against knowing only the overall level of responding. 
Assuming homogeneity of the individual Markov chains, the first-order vs the second-order hypothesis was then tested and rejected at the .01 level for each group (see Suppes \& Atkinson, p. 57, for applying the chi-square test). The obtained values of chi-square, with $36 \mathrm{df}$, were 271,187 , and 71 for Groups 1,2, and 3 , respectively. Individual tests of the second hypothesis were not made because of insufficient data and the relatively large number of rows with empty cells in the individual transition matrices. (While the number of cells in each matrix is 64, the mean number of observations made by $S$ was only 205.) Inspection of the individual second-order transition matrices showed most of them, particularly in Groups 1 and 2, to be highly similar to one another, suggesting rejection of the second null hypothesis also on the individual level. The rejection of the second hypothesis indicates that the prediction is improved even more if the observations on the immediately preceding two trials are known against knowing only the observation made on the single immediately preceding trial.

The order tests can be straightforwardly generalized to a test of $r-1$ order vs $r$ order (Anderson \& Goodman, 1957). We shall not apply further order tests, nor shall we follow the common procedure of deriving parameters, estimating their values, and testing for goodness of fit. The rejection of the second null hypothesis makes it prohibitively hard to derive the Markov model from more basic psychological assumptions and to generate the transition probabilities in terms of one or more psychologically interpretable parameters. For adequate descriptive models one will probably have to look elsewhere.

\section{DISCUSSION}

Although the models tested in this experiment failed to provide a satisfactory account of the data, they nevertheless yield important information regarding the decision-making process operating in sequential search behavior. The procedures devised for comparing actual and optimal search behavior indicated no changes in behavior among problems. The task might be too complicated to allow problem-to-problem leaming, or perhaps $\mathrm{Ss}$ attributed successful performance in the task completely to chance. Another possible reason is that previous experience in similar sequential search problems caused Ss to approach the task with stable patterns of behavior that, because of the relatively small amount of feedback given in the task, were hardly modified.

The comparison of optimal and actual search behavior shows that relative to the optimal policy Ss tended to err in the direction of maximizing detection probabilities rather than in the direction of minimizing the cost per look, and that this error increased in magnitude the larger the differences among the observation costs. To put it differently, Ss strived to achieve a quick detection of the target marble rather than a decrease in the cost per look relative to the optimal solution. As the cost structure changed systematically among groups, causing differences among observation costs to increase, the optimal policy prescribed to look more often in the locations associated with low costs (see Table 2). Ss responded correctly to the changes in the cost structure by observing more frequently the low-cost boxes. However, the frequency of observations in the low-cost boxes was always smaller than predicted by the optimal model.

The bias in favor of maximizing detection probability may be partially explained by assuming that the criterion specified by the instructions was not always adhered to. It may be argued that in addition to minimizing the expected cost of the search Ss were also motivated to end the experiment as fast as possible. Hence the bias in favor of maximizing detection probability. This argument, though it does not explain the differences among the three groups in terms of the discrepancy from optimality, casts doubts on the generalizability of the present results to other
Table 6

One-Stage Transition Probabilities for Each Group

\begin{tabular}{|c|c|c|c|c|c|}
\hline & & \multicolumn{4}{|c|}{ Group 1} \\
\hline & & A & \multicolumn{2}{|c|}{$t$} & $\mathrm{D}$ \\
\hline$t-1$ & $\begin{array}{l}\text { A } \\
\text { B } \\
\text { C } \\
\text { D }\end{array}$ & $\begin{array}{l}.735 \\
.048 \\
.042 \\
.058 \\
\end{array}$ & $\begin{array}{l}.199 \\
.793 \\
.057 \\
.055 \\
\end{array}$ & $\begin{array}{l}.036 \\
.123 \\
.811 \\
.100 \\
\end{array}$ & $\begin{array}{l}.030 \\
.036 \\
.091 \\
.788 \\
\end{array}$ \\
\hline & & A & $\mathrm{B}$ & $\mathrm{C}$ & $\mathrm{D}$ \\
\hline$t-1$ & $\begin{array}{l}\text { A } \\
\text { B } \\
\text { C } \\
\text { D }\end{array}$ & $\begin{array}{l}.678 \\
.080 \\
.033 \\
.041 \\
\end{array}$ & $\begin{array}{l}.210 \\
.712 \\
.086 \\
.057 \\
\end{array}$ & $\begin{array}{l}.056 \\
.153 \\
.758 \\
.096 \\
\end{array}$ & $\begin{array}{l}.057 \\
.056 \\
.123 \\
.807\end{array}$ \\
\hline & & A & B & $\mathrm{C}$ & D \\
\hline$t-1$ & $\begin{array}{l}\text { A } \\
\text { B } \\
\text { C } \\
\text { D }\end{array}$ & $\begin{array}{l}.636 \\
.097 \\
.030 \\
.093\end{array}$ & $\begin{array}{l}.215 \\
.689 \\
.085 \\
.163\end{array}$ & $\begin{array}{l}.060 \\
.150 \\
.743 \\
.264\end{array}$ & $\begin{array}{l}.089 \\
.063 \\
.141 \\
.480\end{array}$ \\
\hline
\end{tabular}

search tasks, where observation costs are considerably higher and minimization of the expected cost of the search may be reasonably assumed to be the sole objective of the decision maker.

The results reported in Tables 4 and 5 might seem inconsistent. While discrepancies from cumulative optimal detection probabilities reported in Table 4 show clearly that $S$ s in Group 3 deviated more from the optimal policy than Ss in Group 2, Table 5 shows that the mean proportion of stepwise optimal decisions for Group 3 (.430) was almost twice as large as that for Group 2 (.227). This inconsistency, however, is only apparent. It has to do with the fact that while the analysis in terms of $y_{v_{w}}$ relates to the mean size of discrepancy from optimal search behavior, the stepwise analysis concerns the number of errors. The decisions in Group 3 that were not stepwise optimal had a much larger effect on increasing the discrepancy from the optimal policy than nonoptimal decisions in Group 2.

In discussing the applicability of the sequential search paradigm investigated in the present study it seems relevant to consider visual search problems, which have received considerable interest recently (Neisser, 1967). In a typical visual search task the $S$ is presented an array of various symbols and asked to scan the array until a target symbol is located (Cowan, 1968). The array can differ in the number of symbols presented, in the field density, and in the size, form, color, and intensity of the symbols. The $S$ is typically asked to minimize search time to target discovery. Since no explicit payoff structure is imposed on the task, prior probabilities of finding the target symbol(s) in specified parts of the array are not manipulated experimentally, and detection probabilities of finding the target symbol in specified parts of the array, if it is there, are typically not assessed, there is no simple way to separate the factors influencing S's sensitivity to the perceptual variables mentioned above from those influencing his attitudes and choice behavior. The two sets of factors are confounded, and one way to separate them is to proceed as in signal detectability research (Green \& Swets, 1966) by introducing costs and probabilities explicitly into the task.

The introduction of costs and probabilities into visual search tasks is complicated by the fact that the array has to be divided into several distinguishable parts, eye movements from one part of the array to another ought to be measured carefully, and 
(subjective) detection probabilities should be assessed precisely. This study adopted a simpler approach by eliminating any perceptual variables from the task and concentrating only on the effects of one variable-observation cost-on S's sequential search behavior. The investigation was concerned only with the decision-making aspects of search behavior. It is hoped that it may also help to clarify the effects of search cost on visual search behavior, and that feasible ways will be found eventually to study the effects of the two sets of factors simultaneously.

A final comment is warranted concerning possible extensions of the sequential search paradigm. A restrictive feature of the task, one that limits its range of applicability, is that the conditional miss probabilities are constant. In real-life sequential search tasks and in visual search problems, independence between successive looks in the same region is usually not maintained. Sequential observations in the same location $i$ are frequently dependent on each other, causing the value of $m_{i}$ to change. Often, if a certain observation in location $i$ fails to detect the target, it suggests that the prevailing detection conditions are poorer than previously expected and that the next observation in the same location will be even more likely to fail. In other words, the values of $m_{i}$ or their estimates by the decision maker may increase monotonically as a function of the number of observations in location i. Other sequential search problems may be considered where values of $m_{i}$ or their estimates decrease in time.

The sequential search paradigm can be extended to the more general case where $m_{i}$ change in time. Supposing $m_{i}$ are constant and the posterior probability of finding the target after $n$ observations in location $i$ is denoted as before by

$$
\mathrm{p}_{\mathrm{i}}(\mathrm{n})=\mathrm{p}_{\mathrm{i}}\left(1-\mathrm{m}_{\mathrm{i}}\right) \mathrm{m}_{\mathrm{i}}^{\mathrm{n}-1}
$$

When $m_{i}$ are not constant, $p_{i}(n)$ is given by

$$
p_{i}(n)=p_{i}\left(1-m_{i n}\right) \prod_{t=1}^{n-1} m_{i t}
$$

where $m_{i t}$ is the conditional miss probability in location $i$ on the $t^{\text {th }}$ observation. Substituting (4) for (3) in (1), the derivation of the optimal policy and the comparison of actual and optimal search behavior may be carried out exactly as before.

\section{REFERENCES}

ANDERSON, T. W., \& GOODMAN, L. A. Statistical inference about chains. Annals of Mathematical Statistics, 1957, 28, 89-110.

BLACK, W. L. Discrete sequential search. Information and Control, 1965, $8,159-162$.

CHEW, M. C., JR. A sequential search procedure. Annals of Mathematical Statistics, 1967, 38, 494-502.

COWAN, T. M. An observing response analysis of visual search. Psychological Review, 1968, 75, 265-270.

GREEN, D. M., \& SWETS, J. A. Signal detection and psychophysics. New York: Wiley, 1966.

KAHAN, J. P., RAPOPORT, A., \& JONES, L. V. Decision making in a sequential search task. Perception \& Psychophysics, 1967, 2, 374-376.

KEMENY, J. G., \& SNELL, J. L. Finite Markov chains. Princeton, N.J.: Van Nostrand, 1960.

MATULA, D. A periodic optimal search. The American Mathematical Monthly, 1964, 71, 15-21.

NEISSER, J. Cognitive psychology. N.Y.: A ppleton-Century-Crofts, 1967.

RAPOPORT, A., \& TVERSKY, A. Cost and accessibility of offers as determinants of optional stopping. Psychonomic Science, 1966, 4, 145-146.

RAPOPORT, A., \& TVERSKY, A. Choice behavior in an optional stopping task. Chapel Hill, N.C.: Psychometric Laboratory Report No.72, January 1969.

SUPPES, P., \& ATKINSON, R. C. Markov learning models for multiperson interactions. Stanford, Calif.: Stanford University Press, 1960.

\section{NOTES}

1. This research was supported by a grant from the Kaplan School of Economics at the Hebrew University of Jerusalem and by Grant No. M-10006 from the National Institute of Mental Health to the University of North Carolina. I wish to thank M. Kubobi and Lyle V. Jones for a critical reading of the manuscript and E. Olmert and N. Vagenberg for assistance in collecting and analyzing the data.

2. Address: Department of Psychology, University of North Carolina, Chapel Hill, North Carolina 27514.

(Accepted for publication March 20, 1969.) 